\title{
SIMULATED X-RAY EMISSION FROM STARBURST DRIVEN WINDS
}

\author{
D. K. STRICKLAND, I. R. STEVENS AND T. J. PONMAN \\ School of Physics $\&$ Astronomy, University of Birmingham, \\ Edgbaston, Birmingham, B15 2TT, U.K.
}

\begin{abstract}
Winds from massive stars and supernovae in starburst galaxies drive global outflows of hot X-ray emitting plasma, as seen in M82 and NGC 253. These galactic winds are important for understanding galaxy evolution \& formation, chemical enrichment of the IGM, and the starburst phenomenon itself.

$\mathrm{X}$-ray observations provide the only direct probe of the hot gas in these winds. However, the limitations of current X-ray observatories and factors such as complex temperature structure, mass loading by ambient material and projection effects all make the link between the observed data and existing 1 \& 2-D modeling and theory difficult to make.

We have therefore begun a program of numerical simulations of galactic winds, concentrating on predicting their observable X-ray properties. We present some initial results, comparing them to the archetypal starburst wind system M82.
\end{abstract}

\section{Introduction}

Starbursts create hot, $10^{6}-10^{8} \mathrm{~K}$, X-ray emitting bubbles of metal enriched gas from thermalised SN ejecta and massive star winds. These superbubbles sweep up and shock heat the ISM in the starburst galaxy, eventually blowing out of the galaxy as galactic winds (c.f. Heckman et al. 1990).

The presence of large scale starburst driven winds in many nearby edgeon galaxies is well established. However, little quantitatively is known about these winds, given the uncertainties in the state and filling factor of the hot gas, and many important questions remain unanswered. 

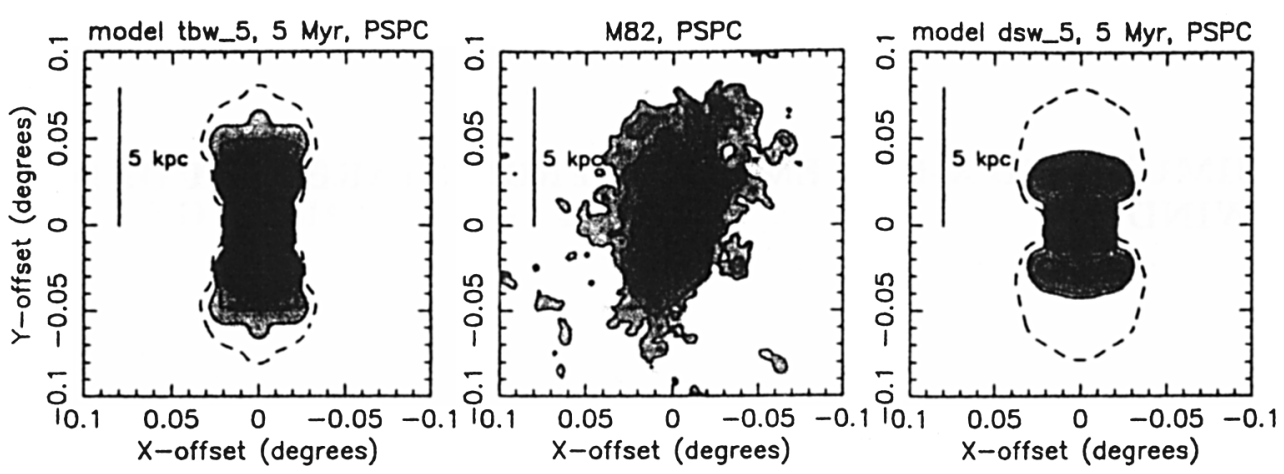

Figure 1. Simulated ROSAT PSPC images for two models at $t=5 \mathrm{Myr}$, shown at the same physical and intensity scale as the PSPC observation of M82. The lowest solid contour is roughly equivalent to a $3 \sigma$ detection on a PSF scale in a $20 \mathrm{ksec}$ exposure. The dashed line shows the true extent of the X-ray emitting volume, as would be seen by an instrument 10 times more sensitive than ROSAT.

\section{Hydrodynamical modelling and selected results}

Previous simulations (c.f. Suchkov et al.1994), while illuminating in regard to some of the processes at work, are very difficult to compare to real observations, and have only studied a limited parameter space. It is impossible to predict the observable consequences of the complex, multi-temperature gas distributions of the simulations, without considering absorption, projection, instrument and spectral fitting effects. All of these must be taken into account before comparing the simulations with real observational data.

We simulate galactic winds using a high resolution 2-D hydrodynamics code, from which we create artificial X-ray observations (e.g. ROSAT, $X M M$ ). These are analysed in the same way a real observation (morphology, temperature structure etc.), allowing a direct comparison between observation and theory.

Fig. 1 compares ROSAT images of two wind models to the PSPC data for M82. The ISM distribution in model tbw_5 was criticized by Suchkov et al. (1994) for creating an artificially high level of collimation for the wind. However, model dsw_5, with the same energy and mass injection but a thinner and non-collimating disk never looks remotely like M82 in the PSPC, as it fades rapidly once the wind blows out of the disk. This demonstrates the need to consider the observable properties of the simulations.

\section{References}

Heckman T. M., Armus L., Miley G. K., 1990, ApJS, 74, 833

Suchkov A. A., Balsara D. S., Heckman T. M., Leitherer C., 1994, ApJ, 430, 511 